\title{
Enhancing web-based learning resources with quizzes through an Authoring Tool and an Audience Response System
}

\author{
Aldo Gordillo, Enrique Barra and Juan Quemada \\ Escuela Técnica Superior de Ingenieros de Telecomunicación \\ Universidad Politécnica de Madrid \\ Avenida Complutense 30, 28040, Madrid, Spain \\ \{agordillo, ebarra,jquemada\}@dit.upm.es
}

\begin{abstract}
Quizzes are among the most widely used resources in web-based education due to their many benefits. However, educators need suitable authoring tools that can be used to create reusable quizzes and to enhance existing materials with them. On the other hand, if teachers use Audience Response Systems (ARSs) they can get instant feedback from their students and thereby enhance their instruction. This paper presents an online authoring tool for creating reusable quizzes and enhancing existing learning resources with them, and a web-based ARS that enables teachers to launch the created quizzes and get instant feedback from the class. Both the authoring tool and the ARS were evaluated. The evaluation of the authoring tool showed that educators can effectively enhance existing learning resources in an easy way by creating and adding quizzes using that tool. Besides, the different factors that assure the reusability of the created quizzes are also exposed. Finally, the evaluation of the developed ARS showed an excellent acceptance of the system by teachers and students, and also it indicated that teachers found the system easy to set up and use in their classrooms.
\end{abstract}

Keywords- quiz; assessment; authoring tool; audience response system

\section{INTRODUCTION}

Web-based learning has become a common practice in education, mostly with the use of Learning Management Systems (LMSs), e-Learning platforms and educational repositories. Quizzes are among the most widely used resources in web-based education. On one hand, self-graded quizzes appropriately integrated into educational resources can help students to check how well they understand the contents and to get to know which parts they should review. On the other hand, graded quizzes and polls are helpful tools for teachers to assess the knowledge of their students of course material as well as to discover the classroom opinions and interests. If teachers use an Audience Response System (ARS) [1] to pose these questions to their students, they can get instant feedback from the class. Based on this feedback, they can offer more detailed explanations to eliminate misconceptions or orchestrate a class discussion.

As a result of these benefits, many authoring tools have been developed to facilitate teachers the creation of quizzes. Nevertheless, these tools are usually limited to create quizzes as independent components, which in most occasions are not easy to integrate with other contents because they are not built conforming to e-Learning standards or use non-appropriate technologies. These limitations may prevent the generated quizzes to be used to enhance existing educational contents or they may considerably reduce their added value. Moreover, although there are some quiz authoring systems that can export the generated questions to standard formats, some of them are not easy to use. For instance, the tools provided by LMSs for creating quizzes are frequently complex and hard to use, and therefore instructors often resort to external applications [2]. Finally, although teachers find a suitable quiz authoring system, employ the created quizzes to enrich existing educational materials is not an easy task. A possible solution to this problem is to use another authoring tool that facilitates this process, enabling teachers to customize, adapt and integrate the quizzes with the rest of the contents. Unfortunately, there is a scarcity of tools of that nature, and thus this approach for building educational contents has not been exploited to its full potential. Taken all this into account, we can state that there is a need for easy to use authoring tools that can be effectively used to enrich existing learning resources with quizzes.

The Virtual Science Hub (ViSH, http://vishub.org) [3] is an e-Learning platform developed as part of the GLOBAL excursion project. Its main contribution is an open source authoring tool for creating Learning Objects (LOs) called ViSH Editor [4]. In this paper we present an enhancement of this tool that allows creating reusable quizzes and enhancing existing materials by adding assessment resources, together with a web-based ARS that enables teachers to get instant feedback from the quizzes. The success of this authoring approach will depend on three main factors: the usability of the authoring tool, the ability of the generated quizzes to be reused and the quality as educational resources of the enriched materials. The success of the ARS will depend on its ease of use and acceptance by teachers and students. Several evaluations and studies were carried out to validate the authoring tool and the ARS based on the aforementioned factors. These evaluations are also described in this paper including their results.

The rest of the paper is organized as follows. The next section reviews related work of quiz authoring tools and ARSs. Section 3 provides an overview of the ViSH Editor authoring 
tool. Section 4 describes the features of the tool to create quizzes and enrich existing materials along with the evaluation of this approach. Section 5 explains the developed ARS together with its evaluation. Finally, section 6 finishes with some conclusions together with an outlook on future work.

\section{RELATED WORK}

An e-Learning authoring tool is an application that enables authors to create complete educational resources by integrating and linking together different objects (such as a picture or a quiz) and custom content generated by them. The major benefits of using authoring tools are cost and time saving, and the facilitation of the creation of high quality courseware. More information about authoring tools can be found in [5] and [6].

Quiz authoring systems are authoring tools designed to create assessment resources. A well known example of these tools is Articulate Quizmaker [7]. Other examples can be found in many LMSs like Moodle or Blackboard, which include a quiz authoring system. Besides, several tools have been developed to replace or enhance these quiz authoring systems [2], [8]. On the other hand, both general and quiz authoring tools are increasingly relying more on the LO approach, focusing on increase the reuse capability of their resources. An example of this is QED (Quiz Engine Developer) [9], an open source quiz authoring system to create standards compliant questions termed "Quiz Objects" due to their similarity to LOs.

Quiz authoring tools not only allow packaging the quizzes into standard e-Learning formats such as SCORM [10] or TIN CAN API [11], they often allow exporting them to question standards. Several question standards exist nowadays. GIFT define questions in simple text files allowing educators to write them using a common text editor. Moodle XML is a Moodle-specific format for importing and exporting questions to be used with the Quiz module. However, it has been accepted as a standard by some e-Learning platforms like Miriada X [12]. Aiken is a question format similar to GIFT but more straightforward. Finally, the most used and recognized standard for the generation of reusable and sharable questions is IMS QTI [13]. The IMS QTI specification describes a data model using XML for the representation of question and test data and their corresponding results reports. It enables the exchange of questions and results data between authoring tools, LMSs, question banks, and e-Learning systems. Quiz authoring systems grounded on the LO approach also allow to tag the quizzes with metadata to improve their reusability and discoverability. The most used LO metadata standard is IEEE LOM [14]. Regarding quiz metadata, [15] points out the scarcity of metadata models dedicated to assessment, and propose an enhanced assessment metadata model for e-Learning operations and an authoring system architecture based on it. Finally, another important feature that quiz authoring tools should provide is multi-device support, since some studies (e.g. [16]) have shown that students prefer to use mobile phones rather than PCs to answer quizzes.

The quantity of available authoring tools for enhancing web-based learning resources with quizzes is significantly lower. One of the most known is Articulate Presenter [17], which allows to integrate quizzes and surveys created through
Articulate Quiz Maker into presentations. Another example is REDEEM [18], an authoring tool for creating Intelligent Tutoring Systems (ITSs) by customizing and enriching existing courseware with questions. Reference [18] shows that REDEEM can be used to create ITSs more effective than the courseware they are based on. Other example is eTEACH [19], an authoring tool for enhancing online video lectures through the embedding of quizzes constructed using a Microsoft Word template and the MathType plugin. Finally, LMSs can be also considered an example of this approach because they allow to enhance e-Learning courses by adding tests.

Audience Response Systems (ARSs), also called Classroom Response Systems (CRSs), allow an entire classroom to respond to questions using remote control devices. The responses are instantly collected and summarized, and can be presented to the class in visual format, usually in chart form. Responses are always anonymous to peers, but some ARSs allow authenticated participation by linking physical devices and users thus allowing teachers to track the students' evolution. Many studies have examined the benefits of using ARSs [1] [20], showing that they can be used to improve student attention [21], [22], engagement [23], [24], and learning performance [22], [24], enhance instruction [21], [22], and stimulate class discussion [21]. There are two different types of ARSs: traditional ARSs and new or rich ARSs. Traditional ARSs are based on the distribution of remote controls (often called "clickers") to the students, which use radiofrequency or infrared signals to communicate with the receiver, which is usually connected to a computer. Students use these devices to answer the questions asked by the teacher. In some cases, the system also provides a desktop application that enables the teacher to handle the poll as well as display the results to students from the computer. An example of this type of ARS is iClicker [25]. Given that these systems use dedicated control devices, the major shortcomings are the high price of the system, the number of question types that can be used (usually restricted to multiple-choice questions), and the lack of interoperability, since controllers and receivers from different companies cannot work between them. The new generation of ARSs allows the use of general purpose devices such as mobile phones instead of proprietary controls. This brings several advantages in comparison with traditional ARSs such as the increment of supported question types and the extension of the available forms of interaction due to the possibility of using touch-based devices [26]. Some of these systems allows participation by sending SMS, such as Poll Everywhere [27]. Other ones use mobile applications for specific platforms like Android or iOS [26]. In this case, the system sends the answers through the Internet connection. Finally, web-based ARSs just require a web browser to connect to the poll and answer the questions. Thereby, any device with a web browser (e.g. PC, mobile phone, tablet, smart TV, etc.) can be used as a remote control. This approach not only allows to provide more interactive and varied quizzes, but also drastically increases the interoperability of the system. There are already some studies that have examined the use of web-based ARSs [28], as well as commercial applications (e.g. [27], [29]) and open source implementations [30]. 


\section{AUTHORING TOOL}

ViSH Editor is a web-based e-Learning authoring tool that aims to facilitate the creation of high quality multi-device LOs. It is open source and the code is available at http://github.com/ging/vish_editor.

The LOs can be combined among them to build more complex ones forming a hierarchy, where the different hierarchy levels are called aggregation or granularity levels. LO models define these levels of granularity and specify how the components can be aggregated as well as the properties of these components [31]. Fig. 1 shows the LO model followed by ViSH Editor, which defines four levels of granularity.

- The first level, which corresponds to the most granular or atomic level, includes raw media files like images, audios or videos and single elements like paragraphs of text or websites. More sophisticated elements such as interactive quizzes or web applications (e.g. SCORM packages) belong to the first level too.

- The second level covers only one LO called "slide" that consists of a collection of level 1 LOs.

- The third level includes any LO that is built as a composition of level 2 LOs (i.e. "slides"). These LOs are identified under the name of "slidesets".

- Finally, the fourth level corresponds to the Interactive Presentation LO. An Interactive Presentation can contain level 3 LOs or directly a slide without using a slideset as a wrapper.

The whole architecture of the system is composed by two main components: the viewer and the editor. The viewer is a fully client-side web application called ViSH Viewer [32] that allows users to view and interact with the created LOs. It is based on HTML5 [33], the new standard for the web. For this reason, any device with a HTML5 compatible web browser can run the tool and view the LOs without any installation being needed. The editor (i.e. ViSH Editor), is the web-based authoring tool where the authors create and edit the LOs. It is composed by two components: the client and the server backend. The client is a HTML5 web application that provides the user interface and most of the features of the editor. The server backend provides functionalities such as file uploading, file conversion (e.g. video and PDF files), exporting and storing. Moreover, ViSH Editor has an integration API to facilitate its integration

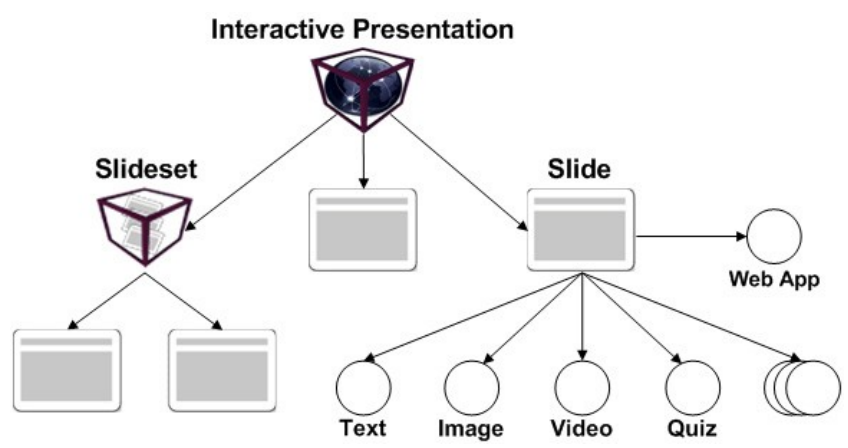

Fig. 1. Learning Object Model of ViSH Editor and/or connection with Learning Object Repositories (LORs), allowing its use in different environments.

ViSH Editor is based on the WYSIWYG (What You See Is What You Get) paradigm and provides a user-friendly interface. It is internationalized and supports several languages such as English, Spanish, French and German. To create a LO, authors can insert many types of resources (e.g. images, videos, documents, websites, SCORM packages, etc.) via its URL, uploading them or by searching on different educational repositories and content providers such as Flickr, YouTube, SoundCloud or LRE [34]. Besides adding resources, authors can type text and create several types of quizzes. ViSH Editor also facilitates authors to fill the metadata of the LOs. All LOs created by ViSH Editor and their metadata are saved in JSON format. However, the LOs can be exported to SCORM and their metadata can be retrieved in IEEE LOM facilitating their integration in LMSs. More details about ViSH Editor can be found in previous publications [4].

To this day, there is a public instance of ViSH Editor accessible through the ViSH e-Learning platform (http://vishub.org) [3]. ViSH is fundamentally formed by a social network, a LOR, a videoconference service, and the ViSH Editor authoring tool that allows ViSH users to create and publish LOs. Both ViSH and ViSH Editor have been developed as part of the GLOBAL excursion European project.

\section{QUIZ AUTHORING AND INTEGRATION}

\section{A. Overview}

ViSH Editor presents the LOs to be created as presentations of slides, on which authors can add new slides and move or remove the existing ones. Each slide is created from a template and inside it authors can create and insert different types of content. The current version allows creating five types of self-graded quizzes:

- Short Answer: requires the student to provide a short response to a statement.

- Multiple Choice: requires to select a single correct choice from a list.

- Multiple Response: requires to select all the correct choices from a list.

- True/False: for each of the statements of a list, requires to select whether it is true or false.

- Sorting: requires to arrange in sequence a list of items.

The quizzes are created using a WYSIWYG rich text editor. Besides, several types of resources such as images, audios or videos can be added to them. Authors can preview the quizzes and the whole presentation from a student's perspective at any time. Fig. 2 shows an example of a listening quiz for English language students created with ViSH Editor. It is composed by several true/false questions, an audio podcast and an image. In this example, students have to listen to the audio podcast and try to answer the questions while they listen. A transcription of the audio is available in subsequent slides, where some words include links to their definition in the Cambridge English Online Dictionary or to some websites that 


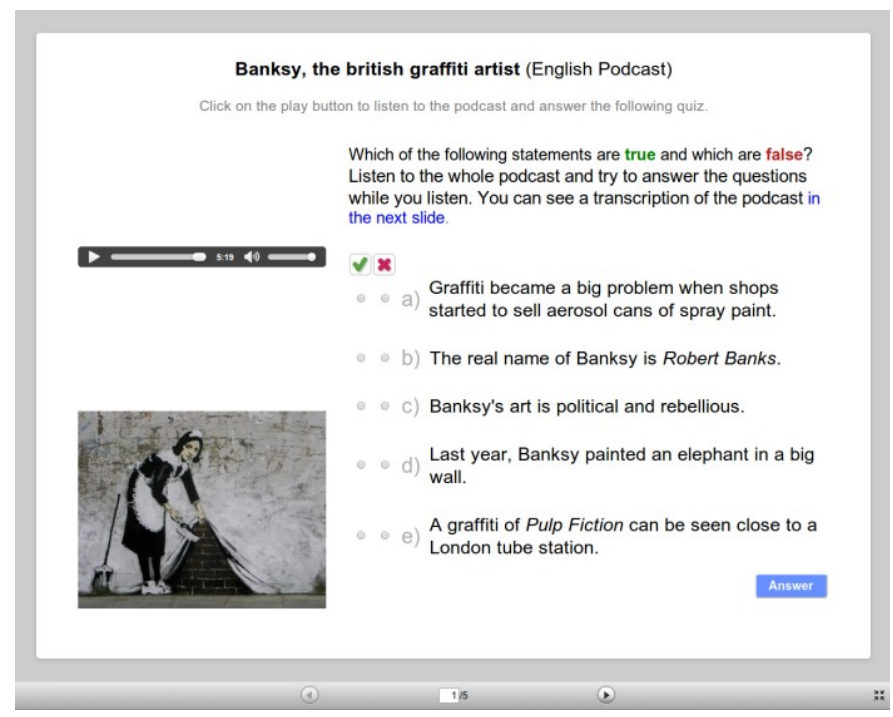

Fig. 2. Banksy, the british graffiti artist (Listening Quiz)

show contents related to the story. Students can access the quiz through any device that runs a HTML5 compliant web browser including mobile phones. This is possible because the audio (and video) files are processed in the server backend after being uploaded in order to be converted to HTML5 compliant formats. This LO may be considered a good example of how to enhance an existing learning material (i.e. the audio podcast) with assessment resources. Fig. 3 shows a screenshot of the ViSH Editor authoring tool when editing the listening quiz.

Besides the possibility of specifying the correct answers, authors can define some settings for the quizzes such as the maximum number of attempts a student has to answer, shuffle choices, or if the quiz should display a button to be launched using the ARS. Moreover, authors can include a lot of information in the metadata (according to the IEEE LOM standard), such as the language, educational context, target age, difficulty, typical learning time, keywords, etc. The created quizzes can be exported to the IMS QTI standard. Furthermore, they can be packaged together with the rest of the contents of the presentation into SCORM packages including their metadata, allowing this way the integration of the enhanced learning resources in LMSs such as Moodle or Blackboard.

In addition to create quizzes from scratch, it is also possible to import IMS QTI questions from XML files and import quizzes from another LOs created with ViSH Editor. Another possibility is to import quizzes from SCORM packages created with other authoring tools (e.g. Articulate Quiz Maker). However, this integration is limited since authors do not have the chance of customize and set up the quizzes unless they use the original tool. Another constraint, which will be removed in future versions of the tool, is that the quizzes added in this way cannot be launched with the ARS.

Finally, the ViSH Editor authoring tool provides some features specifically designed to enrich existing learning resources. Firstly, besides allowing to insert a huge variety of

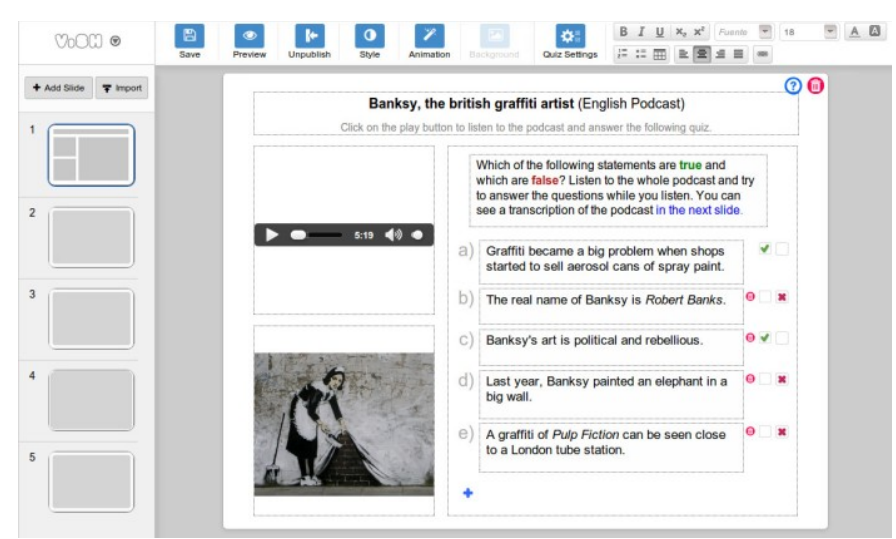

Fig. 3. Editing "Banksy, the british graffiti artist (Listening Quiz)"

resources such as multimedia files, websites, flash objects, documents or SCORM packages, it is possible to convert a PDF presentation (created using Microsoft PowerPoint, LibreOffice or any other authoring system) into a ViSH Editor presentation. This way, static slideshows can be turned into web-based interactive presentations by adding quizzes, videos, web applications and other interactive resources. Secondly, ViSH Editor allows to create other kind of interactive LOs, which can be used to enrich the presentations or individually. An example of this type of LOs are the Enriched Videos, which can be defined as videos augmented with other resources (e.g. quizzes), which can be selected and customized by the author, and that will be displayed automatically when the video reaches a specific point of time or when the student requests one of them explicitly. This feature is quite useful to enrich videos with quizzes, especially in MOOCs [35], since it can be used to enrich video lectures and to automatically track students' progress. An example of an Enriched Video used in a HTML5 MOOC offered by the Universidad Politécnica de Madrid can be seen in Fig. 4. Other interactive LOs that can be created with the current version of ViSH Editor are Flashcards, that allow to enrich infographics, and Virtual Tours, that allow to enrich interactive maps.

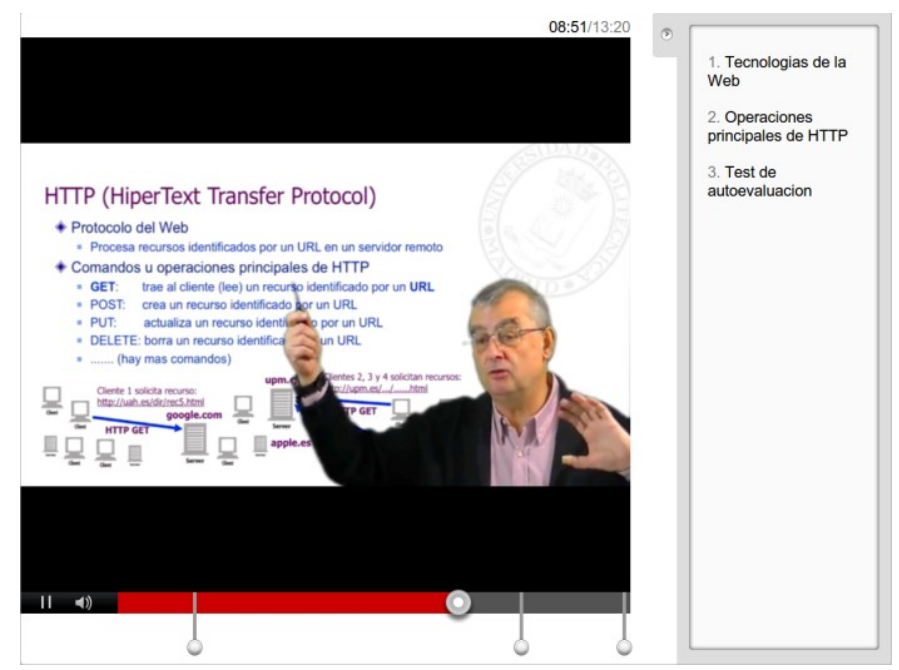

Fig. 4. Enriched Video used in a HTML5 MOOC 


\section{B. Evaluation of the Authoring System}

To evaluate the ViSH Editor authoring tool, a 13 questions survey was conducted to collect authors' feedback on overall opinion, usability and proposed changes among others. ViSH Editor has been used by different types of author: primary and secondary school teachers, lecturers, researchers, student teachers and even high school students and undergraduates. With the aim of evaluating the tool for teachers, students and undergraduates were excluded from the study. An email with the online survey link was sent on December 2013 to all ViSH teachers (as well as student teachers) that had created and published at least one $\mathrm{LO}$ in the ViSH platform during the last three months. A total of 67 surveys were collected. The sample consisted of 67 teachers, 34 males $(50.7 \%)$ and 33 females $(49.3 \%), 19$ to 65 years of age $(\mathrm{M}=35.9, \mathrm{SD}=13.1)$.

Survey results show that $29.9 \%$ of the authors who filled the survey had an "Excellent" overall opinion of ViSH Editor, 61.2\% "Good", 7.5\% "Neutral", 1.5\% "Bad" and that nobody had an "Awful" opinion. The perceived usability was obtained from the question "How would you describe the experience of learning to use ViSH Editor?" in which authors answered on a scale from 1 to 5 with 1 being "very difficult" and 5 being "very easy". This question recorded a mean of 4.2 and a standard deviation of $0.8(\mathrm{M}=4.2, \mathrm{SD}=0.8)$, showing that authors found ViSH Editor easy to use.

Respondents were also asked about their overall experience when using various features of ViSH Editor related to the creation of quizzes and the importation of external learning resources. They answered this question on a scale from 1 to 5 with 1 being "Awful" and 5 being "Excellent". Table I summarizes the results. All features obtained an overall experience rating higher or equal to 3.9 out of 5 with low standard deviation. These data indicate that authors not only found that ViSH Editor is overall easy to use, but also they were satisfied with the specific functionalities to create quizzes and enrich existing learning resources.

TABLE I. AUTHOR'S OVERALL EXPERIENCE RATING (IN A SCALE FROM 1 TO 5 )

\begin{tabular}{|c|c|c|}
\hline Feature & M & SD \\
\hline Creation of quizzes & 3.9 & 0.9 \\
\hline Creation of Enriched Videos & 4.2 & 0.7 \\
\hline Addition of multimedia resources & 4.3 & 0.8 \\
\hline Addition of external websites & 4.4 & 0.7 \\
\hline Importation of slidehows from PDF files & 4.1 & 1.0 \\
\hline Importation of quizzes from external files & 4.2 & 0.9 \\
\hline Importation of other ViSH Editor LOs & 4.5 & 0.7 \\
\hline
\end{tabular}

\section{Evaluation of the Learning Objects}

To evaluate this approach for enriching learning resources we conducted a study to analyze how quizzes influence on the LOs quality. For the study, a total of 209 Interactive Presentations created with ViSH Editor were evaluated and a score based on a quality metric was calculated for each of them. The quality evaluation of the LOs was carried out using a formal LO evaluation model called LORI (Learning Object
Review Instrument) [36]. LORI is one of the better known LO evaluation models and has been tested in a few studies ([37], [38]), which have shown that it can be used to reliably assess some aspects of LOs. In this study, reviewers employed the version 1.5 of LORI, which defines 9 items for LO evaluation related to the following criteria: Content Quality (item 1), Learning Goal Alignment (2), Feedback and Adaptation (3), Motivation (4), Presentation Design (5), Interaction Usability (6), Accessibility (7), Reusability (8), and Standards Compliance (9). When using LORI, reviewers have to rate each item using a 5-point scale and also they can provide comments with their reviews. In this case, they used a web application to conduct the evaluations. The group of reviewers was composed by 15 members: 4 e-Learning professionals, 9 educators and 2 designers. Each LO was evaluated by at least 3 reviewers, generating a total amount of 740 evaluations. A "LORI Weighted Arithmetic Mean (WAM) metric" was used as quality metric. This metric calculates the score of a LO as the weighted arithmetic mean of all LORI items scores, giving different importance to each criterion. The set of weights for the different criteria were obtained through a survey among the reviewers obtaining the following result: $\mathrm{W}_{1}=0.1724$, $\mathrm{W}_{2}=0.1207, \mathrm{~W}_{3}=0.1138, \mathrm{~W}_{4}=0.1414, \mathrm{~W}_{5}=0.1379, \mathrm{~W}_{6}=0.1034$, $\mathrm{W}_{7}=0.0655, \mathrm{~W}_{8}=0.0759$ and $\mathrm{W}_{9}=0.069$, where $\mathrm{W}_{\mathrm{i}}$ corresponds to the weight of the LORI item $\mathrm{i}$. The equation that calculates the LO score takes an input score vector $\left\{i_{1}, \ldots, i_{9}\right\}$, being $i_{i}$ the score of the LORI item $i$ in a scale from 1 to 5 , and yields a single real value in a scale from 0 to 10 according to the following expression:

$$
\begin{gathered}
s\left(\left\{i_{1}, \ldots, i_{9}\right\}\right)=\frac{5}{2} \times \sum_{i=1}^{9} W_{i} \times\left(i_{i}-1\right), s(\{i\}) \in[0,10] \\
\text { where } \sum_{i=1}^{9} W_{i}=1 \text { and } W_{i} \geq 0 \forall i \in\{1, \ldots, 9\}
\end{gathered}
$$

After the evaluation, the 209 Interactive Presentations were grouped depending on the presence or absence of quizzes. Thereby, the first group was formed by presentations that include quizzes $(\mathrm{n}=47)$, and the second group was formed by presentations that do not include quizzes $(n=162)$. After that, both groups were compared with respect to their overall quality score based on the LORI WAM metric, and with respect to their scores on the LORI items (see Table II). Thus, the overall score is expressed in a scale from 0 to 10 , and the scores of the LORI items are expressed in a scale from 1 to 5 . A series of independent samples t-tests were conducted to determine if there were significant differences between the two groups in terms of their scores. In addition, Cohen's d effect size was used to determine the practical significance of differences in overall quality mean scores and LORI items mean scores. When using Cohen's d as a measure of effect size, the 0.2 value indicates a small, 0.5 a medium and over 0.8 a large effect size [39]. The two groups of LOs differed statistically significantly in all LORI items scores as well as overall score. Large effect sizes $(d>0.8)$ were found in the overall score and the first six LORI items. The largest effect size was for the Feedback and Adaptation item $(\mathrm{d}=2.38)$ and the second largest one was for the overall score $(\mathrm{d}=1.30)$. It was expected that the presence of quizzes had a large positive impact on feedback, since quizzes allow to provide feedback concerning the quality or correctness 
TABLE II. OVERALL QUALITY SCORE AND LORI ITEMS SCORES FOR THE TWO GROUPS OF LEARNING OBJECTS

\begin{tabular}{|c|c|c|c|c|c|c|c|}
\hline & \multicolumn{2}{|c|}{$\begin{array}{c}\text { LOs with Quizzes } \\
(n=47)\end{array}$} & \multicolumn{2}{|c|}{$\begin{array}{l}\text { LOs without Quizzes } \\
(n=162)\end{array}$} & \multirow{2}{*}{$\begin{array}{l}\text { Independent } \\
\text { samples t-test } \\
\text { p (2-tailed) }\end{array}$} & \multirow{2}{*}{$\begin{array}{l}\text { Cohen's d } \\
\text { effect size }\end{array}$} \\
\hline & & $M$ & $S D$ & $M$ & $S D$ & & \\
\hline \multicolumn{2}{|c|}{ Overall Score (LORI WAM Quality Metric) } & 7.5 & 1.0 & 5.6 & 1.6 & $<0.0001$ & 1.30 \\
\hline \multirow{9}{*}{$\begin{array}{l}\text { LORI } \\
\text { Items }\end{array}$} & 1. Content Quality & 4.2 & 0.6 & 3.5 & 0.8 & $<0.0001$ & 0.91 \\
\hline & 2. Learning Goal Alignment & 4.2 & 0.5 & 3.4 & 0.9 & $<0.0001$ & 0.96 \\
\hline & 3. Feedback and Adaptation & 3.9 & 0.6 & 2.3 & 0.7 & $<0.0001$ & 2.38 \\
\hline & 4. Motivation & 4.0 & 0.5 & 3.2 & 0.9 & $<0.0001$ & 0.95 \\
\hline & 5. Presentation Design & 3.7 & 0.6 & 3.0 & 0.8 & $<0.0001$ & 0.87 \\
\hline & 6. Interaction Usability & 3.8 & 0.6 & 3.1 & 0.8 & $<0.0001$ & 0.94 \\
\hline & 7. Accessibility & 3.6 & 0.5 & 3.3 & 0.7 & 0.0013 & 0.54 \\
\hline & 8. Reusability & 3.7 & 0.6 & 3.1 & 1.0 & $<0.0001$ & 0.67 \\
\hline & 9. Standards Compliance & 4.6 & 0.4 & 4.4 & 0.4 & 0.0052 & 0.47 \\
\hline
\end{tabular}

of a student's response. It was also expected significant positive impact on overall quality, content quality, motivation and interaction (due to increased interactivity). However, it was not expected that LOs with quizzes had significantly differences (with medium and large effect sizes) in Learning Goal Alignment, Presentation Design, Accessibility, Reusability and Standards Compliance. An explanation for the improvement in the Learning Goal Alignment item is that quizzes may help to clarify the learning goals of the LOs. A possible explanation for the rest of the improvements, as well as for all large effect sizes obtained, is that LOs with quizzes are more likely to have been created by good authors. Therefore, the score differences obtained in this study, may not be only attributable to the presence of quizzes, but also to other factors such as the differences in authors' skills to creating LOs. Anyway, the effect sizes for Feedback and Adaptation and overall quality were significantly larger than the other ones. Given that, we can state that the presence of quizzes is a positive significant factor on LO quality, and therefore the presented approach for enriching learning resources with quizzes is suitable for enhancing LOs.

Finally, the high potential of the created quizzes to be reused in varying e-Learning systems, learning contexts and with learners from differing backgrounds is assured by several factors. Firstly, the quizzes can be customized based on the educator's needs. Secondly, they can be accessed from any device with a HTML5 compliant web browser. Thirdly, they can be tagged with metadata following the IEEE LOM standard. Fourthly, they can be exported to question standards such as IMS QTI. Fifthly, they can be packaged (together with their metadata and other contents) into SCORM packages. Finally, the evaluation results show that LOs with quizzes were positively evaluated in terms of Accessibility $(\mathrm{M}=3.6$, $\mathrm{SD}=0.5), \quad$ Reusability $(\mathrm{M}=3.7, \quad \mathrm{SD}=0.6)$ and Standards Compliance $(\mathrm{M}=4.6, \mathrm{SD}=0.4)$. These results also show that the presence of quizzes did not have a negative impact on reusability, allowing to increase the quality of the LOs without decreasing their ability to be reused.

\section{AUdIENCE RESPONSE SYSTEM}

\section{A. Overview}

This section presents an overview of the web-based ARS that we have developed to enable teachers to launch quizzes created with ViSH Editor. Fig. 5 shows the architecture of the system and the main interactions. First, teachers have to create a LO (or enrich an existing one) with the ViSH Editor online tool and publish it into the ViSH platform. Then, they can access the LO online through the ViSH portal (using the ViSH Viewer web tool), and launch with the ARS any of the quizzes created via ViSH Editor by clicking on a 'Launch' button placed below of them. To use this feature, teachers have to be logged in. When a quiz is launched using the ARS, the ViSH Viewer tool communicates with the ARS API of the ViSH platform to create a new "quiz session". After creating the quiz session, The ARS API responds with the identifier of the quiz session (qid) and a URL to access it (qurl). This is the URL that audience needs to answer the quiz. Once the ViSH Viewer tool receives this response, it pops up a panel displaying the URL to answer the quiz, a QR code which also contains the quiz URL, and some buttons to share the quiz in several social networks such as Twitter, Facebook or Google+. Teachers can display this panel using the classroom projector, allowing the students to copy the URL from the projection screen or to get it from the QR code using their mobile phones. Besides, teachers can use external tools to share the quiz. For instance, they can share the quiz via email or any other messaging system. The students can use any device with a HTML5 compliant web browser to answer the quizzes. When students go to the quiz URL, the ARS API redirects them to the quiz belonging to the quiz session the teacher has launched. The quiz is rendered using also the ViSH Viewer tool, and therefore students see it exactly equal than in the projection screen regardless of the device they are using. That means that students can view and interact with the different elements of the quizzes (e.g. images, audios, videos) as well as answer interactive quizzes (e.g. sorting questions) using their own devices. The answers are always sent anonymously since currently there is no support for authenticated participation. 


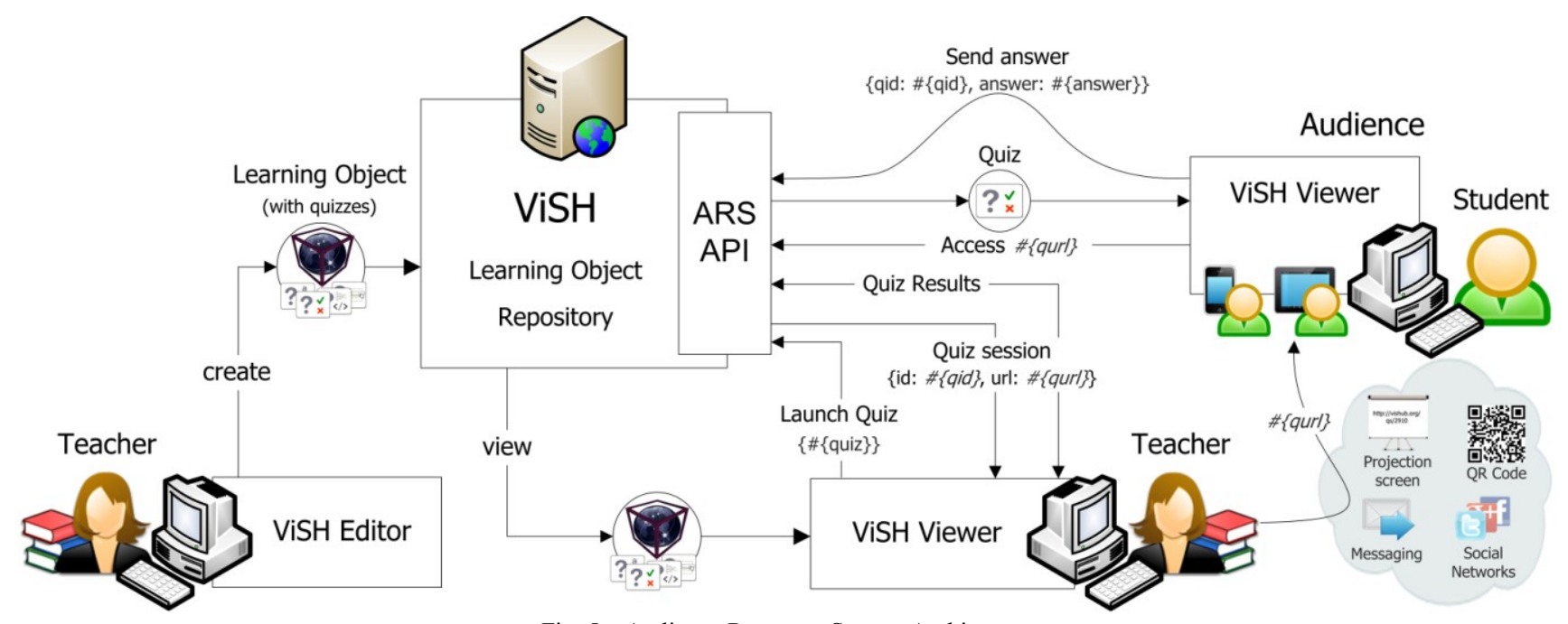

Fig. 5. Audience Response System Architecture

Teachers can also see the results of the quizzes in real time by clicking on a tab in the quiz session panel. The results are presented in chart form (bar graph, pie chart, etc.), where the type of graph depends on the launched quiz. On the other hand, teachers can close a quiz session, preventing it to accept more responses, and save it in the ViSH platform specifying a name. There is panel in the ViSH portal, where teachers can control the state of the quiz sessions (open/closed), view statistics of the responses, view both the quiz that was launched and the LO that included the quiz for a specific session, the dates on which the sessions were opened and closed, etc. The results can be downloaded in XLSX format, so they can be processed using spreadsheet software such as Microsoft Excel or LibreOffice Calc. Next version of the ARS will allow to export the results to IMS QTI.

The major difference of this system in comparison with other web-based ARS is that it is integrated with the authoring tool and thereby it enables to use quizzes integrated in learning resources without using external applications. This way, teachers perform all their actions on the web browser without the need of changing the context.

\section{B. Evaluation}

To evaluate the ARS we conducted a survey to collect feedback from teachers and students who used the system in their classroom on overall opinion, usability and acceptance. Two different questionnaires were used in the survey: one for teachers and one for their students. The ARS was tested in two classrooms by two different teachers. Both teachers were ViSH users familiarized with the ViSH Editor authoring tool who volunteered to participate. A short video tutorial (available in ViSH) about how to use the ARS was provided to them. Then, both teachers prepared a LO including some quizzes to use it in one of their lectures. After each lecture, the teacher and its students filled out the questionnaires. The sample consisted of 2 teachers, both males, one was 31 years old and the other 26, and 21 students, 12 males $(57.1 \%)$ and 9 females $(42.9 \%), 17$ to 30 years of age $(\mathrm{M}=22.5, \mathrm{SD}=3.4)$. Table III summarizes the survey results.
TABLE III. Audience RESPONSE System SuRVEy RESUlts

\begin{tabular}{|l|c|c|c|c|}
\hline \multirow{2}{*}{} & \multicolumn{2}{|c|}{ Teachers } & \multicolumn{2}{c|}{ Students } \\
\cline { 2 - 5 } & $\boldsymbol{M}$ & $\boldsymbol{S D}$ & $\boldsymbol{M}$ & $\boldsymbol{S D}$ \\
\hline $\begin{array}{l}\text { Overall opinion } \\
\text { 1 (Awful) - 5 (Excellent) }\end{array}$ & 4.0 & 0.0 & 4.3 & 0.8 \\
\hline $\begin{array}{l}\text { Value as educational tool } \\
\text { 1 (Worthless) - 5 (Extremely valuable) }\end{array}$ & 4.5 & 0.7 & 4.6 & 0.7 \\
\hline $\begin{array}{l}\text { Ease of setup and use } \\
1 \text { (Awful) - 5 (Excellent) }\end{array}$ & 4.0 & 0.0 & - & - \\
\hline & Yes & No & Yes & No \\
\hline $\begin{array}{l}\text { Would you use the ARS again (in your } \\
\text { lectures)? }\end{array}$ & $100 \%$ & $0 \%$ & - & - \\
\hline $\begin{array}{l}\text { Would you recommend the use of the } \\
\text { ARS to other teachers? }\end{array}$ & $100 \%$ & $0 \%$ & - & - \\
\hline $\begin{array}{l}\text { Would you like this ARS to be used for } \\
\text { other courses too? }\end{array}$ & - & - & $100 \%$ & $0 \%$ \\
\hline
\end{tabular}

These results indicate that both teachers and students had a good overall opinion of the ARS, and that they were very favorable to use it again in the future, denoting a high acceptance of the system. Lastly, teachers considered that the ARS was easy to setup and use in their classrooms.

\section{CONCLUSIONS AND FUTURE WORK}

This paper presents an authoring tool for enhancing existing learning resources by adding reusable quizzes, and a web-based ARS that enables teachers to launch the created quizzes and get instant feedback from the class. The work exposed in this paper show that educators can effectively enhance existing materials by adding quizzes if they are provided with suitable authoring tools. Moreover, they can easily use ARSs to launch these integrated quizzes and improve their instruction.

Given that quizzes have been proven to be able to enhance LO quality, we are going to develop more types of quizzes (e.g. matching, hotspot, fill in the blank, etc.) for future versions of the authoring tool. On the other hand, since the current ARS consists of a module of the ViSH platform, we plan to provide it as an open source interoperable component that can be integrated in different environments. So, a new research line on ARS interoperability standards is opened. Finally, further research is recommended into LO quality indicators as well as instructional strategies to use LOs together with ARSs. 


\section{ACKNOWLEDGMENT}

We wish to acknowledge our gratitude and appreciation to all the GLOBAL excursion project partners, and each one of the project team members, for their contribution to this paper.

\section{REFERENCES}

[1] R. H. Kay and A. LeSage, "Examining the benefits and challenges of using audience response systems: A review of the literature," Computers \& Education, vol. 53, no. 3, pp. 819-827, 2009.

[2] S. Yuuichi, K. Toshihiro, Y. Seisuke, and N. Hiroshi, "Web-based Rapid Authoring Tool for LMS Quiz Creation," in Proceedings of the 7th International Conference on Information Technology Based Higher Education and Training (ITHET'06), 2006.

[3] E. Barra, A. Gordillo, and J. Quemada, "Virtual Science Hub: An Open Source Platform To Enrich Science Teaching," in Proceedings of the International Conference on Educational Sciences and Technology (ICEST 2014), 2014.

[4] A. Gordillo, E. Barra, D. Gallego, and J. Quemada, "An online e-Learning authoring tool to create interactive multi-device learning objects using e-Infrastructure resources," in Proceedings of the 2013 Frontiers in Education Conference (FIE 2013), 2013, pp. 1914-1920.

[5] J. Preclík, "Authoring Tools," in the 9th Annual Conference of Doctoral Students, 2000.

[6] T. Murray, B. Stephen, and S. Ainsworth, Authoring tools for advanced technology learning environments: Toward cost-effective adaptive, interactive and intelligent educational software. Springer, 2003.

[7] “Articulate Quizmaker.”[Online]. Available: http://articulate.com/products/quizmaker.php.

[8] J. R. A. Rodrigues, L. O. Brandäo, M. Nascimento, and P. Rodrigues, "iQuiz: integrated assessment environment to improve Moodle Quiz," in Proceedings of the 2013 Frontiers in Education Conference (FIE 2013), 2013, pp. 13-15.

[9] K. Johnson, T. Hall, and D. O'Keeffe, "Generation of Quiz Objects (QO) with a Quiz Engine Developer (QED)," in Proceedings of the 2005 IEEE International Workshop on Wireless and Mobile Technologies in Education (WMTE 2005), 2005, pp. 5-7.

[10] "Advanced Distributed Learning (ADL), SCORM 2004 4th Edition," 2004. [Online]. Available: http:/www.adlnet.gov/capabilities/scorm/scorm-2004-4th.

[11] J. Poltrack, N. Hruska, A. Johnson, and J. Haag, "The Next Generation of SCORM: Innovation for the Global Force," in Proceedings of the Interservice/Industry Training, Simulation, and Education Conference (I/ITSEC) 2012, 2012, pp. 1-9.

[12] "Miriada X." [Online]. Available: http://www.miriadax.net.

[13] "IMS Question \& Test Interoperability Specification." [Online]. Available: http://www.imsglobal.org/question.

[14] IEEE LTSC, "Draft Standard for Learning Object Metadata (IEEE LOM)," 2002.

[15] W. C. Chang, H. H. Hsu, T. K. Smith, and C. C. Wang, "Enhancing SCORM metadata for assessment authoring in e-Learning," Journal of Computer Assisted Learning, vol. 20, no. 4, pp. 305-316, 2004.

[16] C. Romero, S. Ventura, C. Hervás, and P. De Bra, "An Authoring Tool for Building Both Mobile Adaptable Tests and Web-Based Adaptive or Classic Tests," in Adaptive Hypermedia and Adaptive Web-Based Systems, Springer Berlin Heidelberg, 2006, pp. 203-212.

[17] “Articulate Presenter." [Online]. Available: http://articulate.com/products/presenter.php.

[18] S. Ainsworth and P. Fleming, "Evaluating authoring tools for teachers as instructional designers," Computers in Human Behavior, vol. 22, no. 1, pp. 131-148, 2006.
[19] M. Litzkow and G. Moses, "An Easy to Use Tool for Augmenting Multi-media Lectures with Accessible Self-assessment Exercises," in Proceedings of the 2005 Frontiers in Education Conference (FIE 2005), 2005, pp. 1-6.

[20] C. Fies and J. Marshall, "Classroom Response Systems: A Review of the Literature," Journal of Science Education and Technology, vol. 15, no. 1, pp. 101-109, 2006.

[21] S. W. Draper and M. I. Brown, "Increasing interactivity in lectures using an electronic voting system," Journal of Computer Assisted Learning, vol. 20, no. 2, pp. 81-94, 2004.

[22] J. E. Caldwell, "Clickers in the Large Classroom: Current Research and Best-Practice Tips," Life Sciences Education, vol. 6, no. 1, pp. 9-20, 2007.

[23] R. W. Preszler, A. Dawe, C. B. Shuster, and M. Shuster, "Assessment of the Effects of Student Response Systems on Student Learning and Attitudes over a Broad Range of Biology Courses," Life Sciences Education, vol. 6, no. 1, pp. 29-41, 2007.

[24] R. A. Bartsch and W. Murphy, "Examining the effects of an electronic classroom response system on student engagement and performance," Journal of Educational Computing Research, vol. 44, no. 1, pp. 25-33, 2011.

[25] “iClicker." [Online]. Available: http://www.iclicker.com.

[26] S. Bakrania, "A study on the influence of rich versus traditional classroom response system (CRS) questions on concept retention," in Proceedings of the 2012 Frontiers in Education Conference (FIE 2012), 2012, pp. 1-6.

[27] "Poll Everywhere." [Online]. Available: http://www.polleverywhere.com.

[28] M. Caeiro-Rodriguez, J. Gonzalez-Tato, and M. Llamas-Nistal, "Experiencing a Web-based Audience Response System in engineering lectures," in Proceedings of the 2013 IEEE Global Engineering Education Conference (EDUCON 2013), 2013, pp. 513-519.

[29] “IQPolls.” [Online]. Available: http://iqpolls.com.

[30] M. L. Richardson, "ARSenic: a Free, Open-Source, Web-based Audience Response System Instructor's Guide,” 2013. [Online]. Available: http://uwmsk.org/arsenic.

[31] S. Schluep, "Modularization and structured markup for Web-based Learning Content in an Academic Environment," 2005.

[32] A. Gordillo, E. Barra, and J. Quemada, "Enhancing K-12 science education through a multi-device web tool to facilitate content integration and e-Infrastructure access," in Proceedings of the 7th International Technology, Education and Development Conference (INTED 2013), 2013.

[33] W3C, "HTML5 specification.” [Online]. Available: http://www.w3.org/html/wg/drafts/html/master/Overview.html.

[34] "Learning Resource Exchange (LRE)." [Online]. Available: http://lre.eun.org.

[35] A. Mcauley, B. Stewart, G. Siemens, and D. Cormier, "The MOOC model for digital practice." 2010.

[36] T. L. Leacock and J. C. Nesbit, "A Framework for Evaluating the Quality of Multimedia Learning Resources," Educational Technology and Society, vol. 10, pp. 44-59, 2007.

[37] J. Vargo, J. C. Nesbit, K. Belfer, and A. Archambault, "Learning object evaluation: computer-mediated collaboration and inter-rater reliability," International Journal of Computers and Applications, vol. 25, no. 3, 2003.

[38] F. Krauss and M. Ally, "A Study of the Design and Evaluation of a Learning Object and Implications for Content Development," Interdisciplinary Journal of E-Learning and Learning Objects, vol. 1, pp. 1-22, 2005.

[39] J. Cohen, “A power primer," Psychological bulletin, vol. 112, no. 1, pp. 155-159, 1992. 